\title{
Using the autonomic standards to assess orthostatic hypotension in persons with SCI: a case series
}

\author{
Jill M. Wecht $\mathbb{D}^{1,2} \cdot$ James Wilson $^{3} \cdot$ Jean-Gabriel Previnaire $^{4}$
}

Received: 23 August 2017 / Revised: 19 September 2017 / Accepted: 10 October 2017

(C) International Spinal Cord Society 2017

\begin{abstract}
Introduction Spinal cord injury (SCI) creates a complex and unique syndrome of medical issues related to disruption of somatic and autonomic pathways. Among these impaired control of blood pressure (BP) can significantly impede patients' activities of daily living. The International Standards for the Assessment of Autonomic Function after SCI (ISAFSCI) is used to document the impact of SCI on resting BP (abnormal if below $90 \mathrm{mmHg}$ ) and the presence or absence of orthostatic hypotension $(\mathrm{OH})$, defined as a symptomatic or asymptomatic decrease in $\mathrm{BP}(>/=20 / 10 \mathrm{mmHg})$ upon moving to an upright position.
\end{abstract}

Case presentation Case 1 documents the adverse influence of prescribed medications (antidepressants for neuropathic pain) on $\mathrm{OH}$; case 2 describes the influence of bladder management on cardiovascular instability (autonomic dysreflexia and subsequent $\mathrm{OH}$ ); case 3 describes the association between spasticity and $\mathrm{OH}$; case 4 describes $\mathrm{OH}$ associated with a Valsalva maneuver.

Discussion Impaired control of BP can stem from a combination of medical issues and autonomic dysfunction in persons with SCI. Management strategies for OH will vary depending on the stage of the SCI, the root cause of the $\mathrm{OH}$ and other confounding medical conditions. Non-pharmacological treatment should be considered as a first line of intervention and consideration should be given to cessation of potentially contributory medications prior to implementing pharmaceutical interventions. The systematic use of ISAFSCI by clinicians is recommended to document BP irregularities and to describe the effects of treatment strategies aimed at improving BP control in the SCI population.

\section{Introduction}

The autonomic nervous system (ANS) regulates and maintains cardiovascular homeostasis during routine activities of daily living. Neural activation and withdrawal of parasympathetic and sympathetic cardiovascular influences act to appropriately control heart rate (HR) and blood

Jill M. Wecht

JM.Wecht@va.gov

1 James J Peters VA Medical Center, Room 7A-13, 130 West Kingsbridge Road, Bronx, NY 10468, USA

2 Icahn School of Medicine, Mount Sinai, New York, NY, USA

3 Department of Physical Medicine and Rehabilitation, MetroHealth Rehabilitation Institute of Ohio, Case Western Reserve University, Cleveland, OH, USA

4 Spinal Unit, Centre Calve, Foundation Hopale, Berck-surMer, France pressure (BP). The sympathetic nervous system (SNS) innervates the upper extremity vasculature and the heart from spinal segments T1-T5 via the sympathetic chain ganglia, whereas the vasculature of the splanchnic bed and lower extremities receive SNS innervation from spinal segments T5-L2. The parasympathetic nervous system (PNS) does not directly innervate the vasculature; however, the sinoatrial node is innervated by the vagus nerve (CN X) and acts to rapidly modulate HR. Segmental differences in SNS vascular innervation characterizes the influence of lesion level on cardiovascular responses to orthostasis in individuals with spinal cord injury (SCI) [1]. Interruption of SNS vascular innervation following SCI results in persistent hypotension and bradycardia, with episodic falls in BP during upright positioning, and it is appreciated that presentation of these cardiovascular abnormalities is less prominent in individuals with low cord lesions (i.e., T6 and below). 
In 2009, the International Standards to document remaining Autonomic Function after Spinal Cord Injury (ISAFSCI or Autonomic Standards) defined hypotension as a systolic BP (SBP) below $90 \mathrm{mmHg}$ and bradycardia as a resting $\mathrm{HR}$ below $60 \mathrm{bpm} \mathrm{[2,3].} \mathrm{The} \mathrm{ISAFSCI} \mathrm{also}$ recommends recording the presence or absence of orthostatic hypotension $(\mathrm{OH})$, which is defined by the American Academy of Neurology and the American Autonomic Society as a reduction in SBP $\geq 20 \mathrm{mmHg}$ and/or diastolic BP (DBP) of $\geq 10 \mathrm{mmHg}$ within $3 \mathrm{~min}$ of standing (upright posture), with or without symptoms of orthostatic intolerance (OI) [4]. Although many individuals with SCI demonstrate changes in BP during orthostasis, most do not report symptoms of OI, which are associated with periodic reductions in cerebral perfusion pressure. Commonly reported symptoms associated with OI are light-headedness, dizziness, pre-syncope, and syncope. In addition, nonspecific symptoms of OI include generalized weakness, fatigue, nausea, cognitive slowing, blurred vision, leg buckling, and headache. OI is generally considered a form of ANS dysfunction and is associated with postural tachycardia syndrome and neurally mediated hypotension and may be increased in conditions of chronic disease [5]. The goal of this article is to present a case series of individuals with SCI presenting to a clinic with $\mathrm{BP}$ instability and $\mathrm{OH}$ (or OI) and to document the use of the ISAFSCI during the initial and follow-up assessment visits.

\section{Case presentation}

\section{Case report 1: OI related to medication for neuropathic pain}

A 60-year-old male in France incurred a vertebral burst fracture resulting in T3 AIS A paraplegia following a motor vehicle collision in September 2013. The patient was admitted to the intensive care unit for 1 month following injury for care and invasive ventilation with tracheostomy. In November, the patient was transferred to the rehabilitation unit where he experienced episodic $\mathrm{OH}$ (BP 70/40 $\mathrm{mmHg}$ ) during transfers, which improved over time with application of compression stockings and abdominal binding, and his resting SBP was stable at $120 \mathrm{mmHg}$. In February 2014, the tracheostomy, compression stockings, and abdominal binding were removed. The patient reported abdominal pain and chronic constipation with severe neuropathic pain. In March 2014, trans-anal irrigation was recommended for the constipation and abdominal pain and the patient was started on gabapentin, which was ineffective at treating the neuropathic pain. Pregabalin (300 mg BID) and duloxetine (60 mg once daily) resulted in only a slight improvement in pain symptoms. Amitriptyline was added and titrated to $50 \mathrm{mg}$ once daily, which was very effective in controlling the patient's pain. One week later the patient experienced syncope (BP $60 / 40 \mathrm{mmHg}$ ) while performing trans-anal irrigation, and after 3 days, syncope occurred again at lunchtime while seated in his wheelchair. Compression stockings and an abdominal binder were applied and patient was started on midodrine hydrochloride $7.5 \mathrm{mg}$ TID. The following day the patient experienced syncope (BP 60/30 mmHg) while showering. Amitriptyline was stopped and the syncopal episodes resolved within a few days; however, his neuropathic pain worsened. At $10 \mathrm{mg}$ once daily, amitriptyline was ineffective but at $25 \mathrm{mg}$ once daily syncopal episodes returned so amitriptyline was continued at $10 \mathrm{mg}$ twice daily as needed and the patient has not reported a subsequent syncopal incident. In conclusion, the initial ISAFSCI classification for autonomic control of $\mathrm{BP}$ was abnormal (presence of $\mathrm{OH}$ but normal resting $\mathrm{BP}$ ) and the final classification, after the intervention, was normal.

\section{Case report 2: $\mathrm{OH}$ association with bladder hyper- reflexia}

In August 2013, a 17-year-old male in France experienced a fracture of his C6 vertebra following a diving accident, resulting in spastic C7 AIS A tetraplegia. On day 7 of his rehabilitation stay compression stockings and an abdominal binder were worn and his resting SBP was $100 \mathrm{mmHg}$ (i.e., normal); however, the patient experienced daily bouts of symptomatic $\mathrm{OH}$ (BP 60/30 $\mathrm{mmHg}$ ) during the morning transfer to his wheelchair. The initial ISAFSCI classification for autonomic control of BP was abnormal (presence of $\mathrm{OH}$ with normal resting $\mathrm{BP})$. The patient was started on midodrine (5 mg BID: 7:00 a.m. and 12:00 p.m.), but he continued to experienced moderate $\mathrm{OH}$, without syncope. In November, the patient struggled with episodic autonomic dysreflexia (AD) secondary to bladder hyper-reflexia, and worsening OI. The dose of midodrine was increased $(10 \mathrm{mg}$ BID), his anticholinergic dosing was increased (oxybutynin $5 \mathrm{mg}$ TID), and trospium chloride was added (20 mg BID). Although the patient continued to experience episodic AD and $\mathrm{OH}$, there were slight improvements in symptoms. In February 2014, the patient underwent detrusor botulinum toxin injection, which resulted in abolition of $\mathrm{AD}$ and $\mathrm{OH}$; the patient was able to discontinue midodrine treatment and remove compression stockings and abdominal binding. The final ISAFSCI evaluation for autonomic control of BP was normal.

\section{Case report 3: $\mathrm{OH}$ exacerbated by antispasticity medications}

In May 2017, a 68-year-old male in the United States suffered a low speed motor vehicle collision and his initial 
workup revealed traumatic brain injury and C3 AIS C SCI requiring a $\mathrm{C} 3-7$ laminoplasty. His acute hospital course was further complicated by persistent bradycardia and hypotension. A dual chamber cardiac pacer was implanted 2 weeks into his admission. Three weeks after his accident he was transferred to acute inpatient rehabilitation where his resting BP averaged 130/70 $\mathrm{mmHg}$ (i.e., normal); however, his therapy time was limited due to symptomatic OH (BP $60 / 40 \mathrm{mmHg}$ ), which resulted in a brief return to the acute care hospital. His $\mathrm{OH}$ was relatively well controlled with compressive stockings, abdominal binder and midodrine (10 mg TID). Six weeks after injury he developed increased spasticity in his right shoulder and baclofen was started with serial ISAFSCI assessments. Baclofen was initiated at 10 mg QHS and his blood pressure remained stable in therapy. When baclofen was increased to $10 \mathrm{mg}$ TID, the patient again demonstrated symptomatic $\mathrm{OH}$ (BP $83 / 50 \mathrm{mmHg}$ ) and was tapered down to $5 \mathrm{mg}$ QHS. Even at the lower dose of baclofen, $\mathrm{OH}$ continued to limit his progress in therapy so fludrocortisone $0.1 \mathrm{mg}$ daily was added. By the time of discharge, symptomatic hypotensive episodes were rare and his spasticity was adequately controlled.

\section{Case report 4: $\mathrm{OH}$ associated with Valsalva maneuver}

In May 2016, a 35-year-old male in France was involved in a motor vehicle collision and incurred a fracture of the T4 vertebra, as associated with T2 AIS A spastic paraplegia. He was prescribed intermittent catheterization five times a day, with anticholinergics (trospium chloride (20 mg BID)). During his sub-acute hospitalization episodes of $\mathrm{OH}$ and $\mathrm{AD}$ were rare and were not reported during the chronic phase of injury, and his resting SBP remained stable at 110 $\mathrm{mmHg}$ (i.e., normal). Four months after his injury and before discharge, the patient took part in a clinical study on autonomic testing with the cold pressor test (CPT) and the Valsalva maneuver [6]. The CPT triggered moderate AD (SBP: from 100 to $150 \mathrm{mmHg}$ ), which was accompanied by headache and bradycardia (HR: $55 \mathrm{bpm}$ ). The Valsalva maneuver was performed in the seated position, in which the patient was asked to maintain an expiratory mouth pressure of approximately $30 \mathrm{mmHg}$ for $15 \mathrm{~s}$ (if possible) via a bugle with an air leak. During the Valsalva maneuver there was a continuous drop in SBP from 100 to $65 \mathrm{mmHg}$ in less than $5 \mathrm{~s}$, a concomitant increase in HR from 60 to 70 $\mathrm{bpm}$. The patient reported feeling dizzy and discontinued the expiratory challenge, resulting in spontaneous recovery of SBP within $30 \mathrm{~s}$, which was facilitated by raising his legs. The maneuver was repeated 5 min later with a mouth pressure of $15 \mathrm{mmHg}$, resulting in an asymptomatic drop in SBP (100 to $80 \mathrm{mmHg}$ ), which was classified as $\mathrm{OH}$. At discharge, the ISAFSCI for autonomic control of resting BP was classified normal, although $\mathrm{OH}$ and $\mathrm{AD}$ were present during provocation.

\section{Discussion}

$\mathrm{BP}$ instability and $\mathrm{OH}$, which are present in many individuals with SCI, can be disabling, and can stem from a combination of medical issues including low plasma volume, hyponatremia and cardiovascular deconditioning. In addition, ANS dysfunction resulting in impaired SNSmediated vasoconstriction may lead to venous pooling, decreased preload and stroke volume. The prevalence of $\mathrm{OH}$ following SCI may be as high as $74 \%$, although symptoms are less common in incomplete injuries, especially below $\mathrm{T} 6$ [5]. $\mathrm{OH}$ tends to improve with time given several factors including activation of renin-angiotensin aldosterone system [7, 8], alterations in the sensitivity of the baroreceptor reflex [9], and atrophic changes in lower extremity venous vascular smooth muscle tone [10, 11]. Furthermore, symptoms may improve independent of increased systemic BP, suggesting adaptation in autoregulation of cerebral blood flow [12].

In 2010 recommendations were published in the form of a basic cardiovascular data set for use in the collection of clinical data pertaining to $\mathrm{BP}$ and $\mathrm{OH}$ [13], which include prior history, medication use, and postural position of the patient during the BP assessment. Once resting hypotension and/or $\mathrm{OH}$ has been established based on the Autonomic Standards criteria, further workup for potential causes and/ or exacerbating contributors should be recorded with the SCI Data Sets based on a thorough medical history and physical examination. Symptoms should be correlated with activity and time of day and routine blood work can be considered to rule out factors not directly related to autonomic dysfunction, including anemia, dehydration, infection, or renal impairment. More advanced diagnostics can also be considered. New onset of $\mathrm{OH}$ during the sub-acute and chronic phases of SCI may also raise concerns for posttraumatic syringomyelia, necessitating magnetic resonance imaging. Provocative maneuvers and battery of tests might be helpful to detect those patients at risk of $\mathrm{OH}$.

Management strategies for $\mathrm{OH}$ will vary depending on the stage of the SCI (acute or chronic), the presence and severity of symptoms, individual responses to medical intervention, and confounding medical conditions. Nonpharmacological treatment should be considered as a first line of intervention and include eating small meals, liberalizing fluid and salt intake, elevating the head of the bed overnight, donning of abdominal binder and compressive stockings, avoiding rapid positional changes, and lowerextremity functional electrical stimulation. These interventions should be tailored to the individual patient and may be 
used in combination as needed, because to date no single treatment has been shown reliably effective.

Pharmacological treatment strategies should begin by stopping potentially exacerbating medications such as antidepressants, antihypertensives, antispasmodics, anticholinergics, diuretics, and narcotics. Next clinicians can consider prescribing medications such as fludrocortisone, ephedrine, and midodrine. Fludrocortisone is a corticosteroid with a primarily mineralocorticoid effect to increase renal sodium retention, ephedrine is a nonselective sympathomimetic, and midodrine is an alpha-1 agonist which causes venous and arterial constriction. Midodrine has been approved by the Food and Drug Administration (US-FDA) since 1996 for treatment of symptomatic neurogenic $\mathrm{OH}$ and is the most widely prescribed anti-hypotensive agent in the SCI population [6]. Although several of these pharmacological interventions are illustrated in the cases above, it should be noted that proof of the efficacy for use to improve orthostatic BP responses in the SCI population is limited [14]. In 2014, the FDA approved droxidopa for use in treatment of symptomatic neurogenic $\mathrm{OH}$; however, wide spread accessibility to droxidopa is limited and this agent has not been formally tested for safety and efficacy for use in the SCI population [7].

SCI creates a complex and unique syndrome of medical issues. Common considerations include motor paralysis and weakness along with numbness and pain owing to loss of motor and sensory neural control; however, perhaps less prominent autonomic impairments can be similarly disabling. Among these $\mathrm{OH}$ can significantly impede a patient's tolerance of upright activities, essentially limiting their independence and their return to activities of daily living. The ISAFSCI assessment tool is a starting point for gauging the impact of SCI on autonomic control of systemic cardiovascular function, which was initially published in 2009 [3], and is primarily based on expert opinion as well as input from the international community of SCI clinicians [8, 9]. In 2017, inter-rater reliability was found to be moderate to strong for various sections on the ISAFSCI [10] and providers and students are encouraged to complete an online training course entitled: Autonomic Standard Training E-Program (http://asia.learnshare.com/) [11], which was designed to facilitate assessment of remaining ANS function and to remind clinicians of the importance of understanding, diagnosing and treating autonomic impairments, and to track the effectiveness of interventions aimed at improving organ system function post-SCI.

\section{Points for consideration}

The original intention was that the ISAFSCI should be used by clinicians during the initial clinical assessment to determine a patient's prior history and likelihood of future struggles with issues pertaining to ANS dysfunction. It was with additional intent that the ISAFSCI be used in conjunction with the SCI Data Sets to document the likelihood of ANS dysfunction (ISAFSCI) and the effect of treatment strategies to improve overall organ-system function (SCI Data Sets). However, current developments and discussions have brought to light the need for open dialog from the SCI community regarding the use and utility of the ISAFSCI, which is presently being assessed for modification and revision.

Related to these case studies documenting $\mathrm{OH}$ and the use of the ISAFSCI, it is important to stress that $\mathrm{OH}$ is not a permanent condition and the impact of other dysautonomias should be considered prior to introducing pharmacologic interventions to treat $\mathrm{OH}$ [15]. Although symptomatic $\mathrm{OH}$ is commonly found during the acute phase following injury, symptoms often resolve with time and proper management. Moreover, in its current form, the ISAFSCI only documents history of $\mathrm{AD}$, rather than the presence of $\mathrm{AD}$ at the time of clinical assessment. In these cases, the ISAFSCI can be used to document the history of both $\mathrm{AD}$ and $\mathrm{OH}$ and the likelihood that a patient may continue to struggle with $\mathrm{BP}$ regulation, so that therapeutic strategies (such as pain management, case report 1) can be adapted. On the other hand, the effectiveness of interventions aimed at "normalizing" end-organ function, with implication to autonomic regulation, should also be monitored using the Data Sets (case reports 1-3). These issues should be clarified in version 2 of the ISAFSCI.

Acknowledgements This project was supported, in part, by the Department of Veterans Affairs, Veterans Health Administration, Rehabilitation Research and Development Service Center for the Medical Consequences of Spinal Cord Injury (grant nos. B9212-C, B2020-C).

\section{Compliance with ethical standards}

Conflict of interest We certify that no party having a direct interest in the results of the research supporting this article has or will confer a benefit on us or on any organization with which we are associated and, if applicable, we certify that all financial and material support for this research (e.g., NIH or NHS grants) and work are clearly identified in the acknowledgements.

\section{References}

1. Krassioukov A, Claydon VE. The clinical problems in cardiovascular control following spinal cord injury: an overview. Prog Brain Res. 2006;152:223-9.

2. Krassioukov A, Biering-Sorensen F, Donovan W, Kennelly M, Kirshblum S, Krogh K, et al. International standards to document remaining autonomic function after spinal cord injury. J Spinal Cord Med. 2012;35:201-10.

3. Alexander MS, Biering-Sorensen F, Bodner D, Brackett NL, Cardenas $\mathrm{D}$, Charlifue $\mathrm{S}$, et al. International standards to 
document remaining autonomic function after spinal cord injury. Spinal Cord. 2009;47:36-43.

4. Freeman R, Wieling W, Axelrod FB, Benditt DG, Benarroch E, Biaggioni I, et al. Consensus statement on the definition of orthostatic hypotension, neurally mediated syncope and the postural tachycardia syndrome. Auton Neurosci. 2011;161:46-8.

5. Frith J, Ng WF, Day CP, Payne B, Sheerin N, Gorman G, et al. Orthostatic intolerance is common in chronic disease--a clinical cohort study. Int J Cardiol. 2014;174:861-3.

6. Previnaire JG, Soler JM, Leclercq V, Denys P. Severity of autonomic dysfunction in patients with complete spinal cord injury. Clin Auton Res 2012;22:9-15.

7. Mathias CJ, Christensen NJ, Corbett JL, Frankel HL, Goodwin TJ, Peart WS. Plasma catecholamines, plasma renin activity and plasma aldosterone in tetraplegic man, horizontal and tilted. Clin Sci Mol Med. 1975;49:291-9.

8. Mathias CJ, Christensen NJ, Frankel HL, Peart WS. Renin release during head-up tilt occurs independently of sympathetic nervous activity in tetraplegic man. Clin Sci (Lond) 1980;59:251-6.

9. Phillips AA, Krassioukov AV, Ainslie PN, Warburton DE. Baroreflex function after spinal cord injury. J Neurotrauma 2012;29:2431-45.
10. Wecht JM, de Meersman RE, Weir JP, Bauman WA, Grimm DR. Effects of autonomic disruption and inactivity on venous vascular function. Am J Physiol Heart Circ Physiol 2000;278:H515-20.

11. Wecht JM, de Meersman RE, Weir JP, Spungen AM, Bauman WA. Cardiac homeostasis is independent of calf venous compliance in subjects with paraplegia. Am J Physiol Heart Circ Physiol. 2003;284:H2393-9.

12. Handrakis JP, DeMeersman RE, Rosado-Rivera D, LaFountaine MF, Spungen AM, Bauman WA, et al. Effect of hypotensive challenge on systemic hemodynamics and cerebral blood flow in persons with tetraplegia. Clin Auton Res. 2009;19:39-45.

13. Krassioukov A, Alexander MS, Karlsson AK, Donovan W, Mathias CJ, Biering-Sorensen F. International spinal cord injury cardiovascular function basic data set. Spinal Cord. 2010;48:586-90.

14. Wecht JM, Bauman WA. Implication of altered autonomic control for orthostatic tolerance in SCI. Auton Neurosci. doi:10.1016/j. autneu.2017.04.004.

15. Salim MS, Mazlan M, Hasnan N. Intracerebral haemorrhage following uncontrolled autonomic dysreflexia post suprapubic catheter placement surgery. Spinal Cord Ser Cases. 2017;3:17043. 